\title{
Autoregressive Order Selection for Rotating Machinery
}

\author{
Suguna Thanagasundram ${ }^{\dagger}$ and Fernando Soares Schlindwein ${ }^{\dagger}$ \\ Department of Engineering, University of Leicester, University Road, Leicester LE1 7RH, UK
}

(Received 6 October 2005; accepted 17 May 2006)

\begin{abstract}
This paper provides a practical rule for determining the minimum model order for Autoregressive (AR) based spectrum analysis of data from rotating machinery. The use of parametric methods for spectral estimation, though having superior frequency resolution than Fast Fourier Transform (FFT) based methods, has remained less favoured mainly because of the difficulties in estimating the model order. The minimum model order $p_{\text {min }}$ required is the ratio of the sampling rate and the rotating speed of the machine. This is the number of samples in one shaft revolution. Traditional model order selection criteria, Akaike Information Criterion (AIC), Final Prediction Error (FPE), Minimum Description Length (MDL), Criterion Autoregressive Transfer-function (CAT), and Finite Information Criterion (FIC) are used to estimate the optimal order. These asymptotic criteria for model order estimation are functions of the prediction error and the optimal order of an AR model is chosen as the minimum of this function. Experimental results, using vibration data taken from a dry vacuum pump at different sampling rates and rotating speeds, show that at $p_{\min }$ there is a marked reduction in the prediction error. For low speed rotating machinery, the optimal order is $p_{\min }$. As the speed of the rotating machine increases, there is some advantage in using twice or thrice $p_{\min }$, to produce more accurate frequency estimates. The BoxJenkins method of order determination using autocorrelation and partial autocorrelations plots are also used for justification of the selection of this minimal order.
\end{abstract}

${ }^{\dagger}$ Member of the International Institute of Acoustics and Vibration (IIAV)

\section{INTRODUCTION}

Fault detection and prognosis of equipment is an established technique in many industries worldwide today. Much of the equipment monitored, such as pumps, diesel engines, compressors, and electric motors, belong to the class of rotating machines and produce signals that are quasi-stationary time series. The reliability, availability, and maintainability of these machines are critical to the overall performance and operation of many companies for maintaining their competitiveness in a global marketplace. It is clearly important to receive warnings of problems before failure and outage occurs. Rotating machinery produce signals that are random processes. Examples of these are displacement measurements from proximity probes, vibrations from accelerometers, sound from acoustic emission sensors, and pressure signals from calibration gauges. Signals captured from rotating machines can be used as indicators of the equipment's health for fault prediction and prevention. One way of achieving this is to use time domain analysis, ${ }^{1}$ where statistical parameters are computed and compared with baseline figures, as a change in these parameters may indicate imminent malfunction. An alternative approach is using frequency domain analysis, ${ }^{2}$ where the spectra of the faulty signals are compared with a baseline spectrum obtained from machinery run in normal no-fault conditions. ${ }^{3,4}$

Power Spectral Density (PSD) estimation is performed predominantly using classical techniques based on the Fast Fourier transform (FFT). The FFT is the favoured method for spectral analysis as it is well established and there are Commercial-Off-The-Shelf (COTS) products which aid the implementation of tools for frequency estimation of signals as part of larger condition monitoring programs for fault de- tection in machines. An alternative class of frequency estimation methods is parametric modelling. The parametric approach is based on modelling the signal under analysis as a realisation of a particular stochastic process and estimating the model parameters from its samples. These methods are commonly used in seismic analysis, stock market forecasting, and in biomedical engineering. ${ }^{5}$ The usage of parametric spectral analysis for fault detection and condition monitoring of rotating machinery has remained low, mainly because the order of parametric models has to be determined beforehand to obtain good frequency estimates.

In recent years, there have been some investigators who have applied the technique of parametric modelling for condition monitoring investigations. Mechefske has employed parametric modelling for fault detection of bearing faults. ${ }^{6-8}$ Mechefske has noted that AR modelling is especially useful in low speed machinery, as recording long periods of data in slow speed machines is impractical and the AR method is particularly adequate in such cases, as it can work with short data records and achieve better resolution than the FFT method. He has acknowledged that finding the model order is the most critical step in parametric modelling and has to be accurately determined for power spectral density approximations. Dron ${ }^{9-12}$ has studied the usage of an AR modelling for vibration analysis of a forming press for a conditional maintenance program. He has noted that parametric methods are particularly worthwhile in the early detection of faults, especially when two typical frequencies are close to each other. $\mathrm{He}$ also has acknowledged that the model order selection is one of the major problems encountered when implementing parametric spectrum analysis methods.

In a recent work in 2001, Wenyi Wang has effectively applied the Minimum Phase AutoRegressive (MPAR) approach 